\title{
Combined strategy of Waterston shunt percutaneous occlusion and medical treatment with sildenafil for management of pulmonary hypertension in an adult patient with corrected tetralogy of Fallot
}

\author{
Giancarla Scalone ${ }^{1,2}$, Omar Gomez-Monterrosas ${ }^{2,3}$, Roland Fiszer ${ }^{4}$, Małgorzata Szkutnik ${ }^{5}$, Michat Gałeczka ${ }^{5}$, \\ Jacek Białkowski ${ }^{5}$ \\ ${ }^{1}$ Cardiology Department, Catholic University of Sacred Heart, Rome, Italy \\ ${ }^{2}$ Cardiology Department, Dietrich-Bonhoeffer Klinikum Neubrandenburg, Neubrandenburg, Germany \\ ${ }^{3}$ Cardiology Department, Thorax Institute, IDIBAPS, Institut d'Investigacions Biomediques Agust Pi i Sunyer, Hospital Clinic, Barcelona, Spain \\ ${ }^{4}$ Department of Congenital Heart Diseases and Pediatric Cardiology, Silesian Center for Heart Diseases, Zabrze, Poland \\ ${ }^{5}$ Department of Congenital Heart Diseases and Pediatric Cardiology, Silesian Center for Heart Diseases, Medical University of Silesia, \\ Katowice, Poland
}

Adv Interv Cardiol 2017; 13, 3 (49): 277-278

DOI: https://doi.org/10.5114/aic.2017.70204

A female patient with diagnosis of severe tetralogy of Fallot (TOF) underwent a palliative Waterston shunt (WS) at 5 months of age [1]. Three years later, corrective surgery was performed in another hospital. The surgical report stated closure of the WS. The patient remained in NYHA II and had two successful pregnancies. At the age of 38 years, she was admitted to our hospital for worsening of symptoms (NYHA III). The computed tomography (CT) angiogram showed patency of the WS (Figures $1 \mathrm{~A}$ and $\mathrm{B}$ ). At the right heart catheterization, the pressures before and after the nitric oxide test were $79 / 39 / 54 \mathrm{~mm} \mathrm{Hg}$ and 73/35/48 $\mathrm{mm} \mathrm{Hg}$ in the pulmonary artery (PA), and 125/66/89 and 140/65/94 $\mathrm{mm} \mathrm{Hg}$ in the aorta (Ao), respectively. Moreover, the pressure gradient of the right pulmonary artery (RPA) close to the WS was $16 \mathrm{~mm} \mathrm{Hg}$, whereas the proximal and distal PA pressures to the shunt were $79 / 39 / 54$ and $63 / 36 / 49$, respectively. Pulmonary vascular resistance (PVR) was 2.2 Wood units (WU), and pulmonary to systemic flow ratio calculated by the Fick formula (Qp/Qs) was 3.1 Moreover, the occlusion test with a TyShak balloon was positive. The patient was treated with $75 \mathrm{mg}$ of sildenafil [2] for 6 months, obtaining a PA pressure of 78/37/52 $\mathrm{mm} \mathrm{Hg}$, an Ao pressure of $125 / 66 / 89 \mathrm{~mm} \mathrm{Hg}$, and PVR of $2.6 \mathrm{WU}$ in the next cardiac catheterization. For these reasons, we decided to occlude the shunt with a $12 \mathrm{~mm}$ Amplatzer muscular ventricular septal defect occluder (MVSO) (Figure 1 C, yellow asterisk) from the arterial side, achieving a reduction of PA trunk and left PA pressure, with a value of 62/28/41 $\mathrm{mm} \mathrm{Hg}$. The patient remained asymptomatic until 43 years old, when she was hospitalized due to atrial fibrillation, successfully treated with ablation [3]. The right catheterization showed a PA pressure of $45 / 20 / 30 \mathrm{~mm} \mathrm{Hg}$, an Ao pressure of $125 / 84 \mathrm{~mm} \mathrm{Hg}$, and a complete closure of the WS (Figure $1 \mathrm{D}$ ). The clinical worsening of the patient, and our positive experience with sildenafil for the treatment of elevated pulmonary pressure in a child after the Glenn procedure [4], represented the principal reasons for the shunt closure and subsequent sildenafil therapy. In our opinion, the MVSO with symmetrical retention discs is able to reduce the risk of embolization in case of pulmonary hypertension. Another option of treatment could be implantation of a covered stent in the pulmonary artery to occlude the WS; indeed, an additional advantage of this therapy is the dilation of the stenosed RPA. Our case shows how a combined strategy of medical treatment with sildenafil and percutaneous occlusion of the residual aortic-pulmonary shunt may be useful for the management of pulmonary hypertension in a patient with corrected TOF.

\section{Corresponding author:}

Giancarla Scalone MD, PhD, Cardiology Department, Catholic University of Sacred Heart, Largo Agostino Gemelli 8, 00168 Rome, Italy, phone: +39 3334943216, e-mail: gcarlascl@gmail.com

Received: 18.05.2017, accepted: 30.07.2017. 

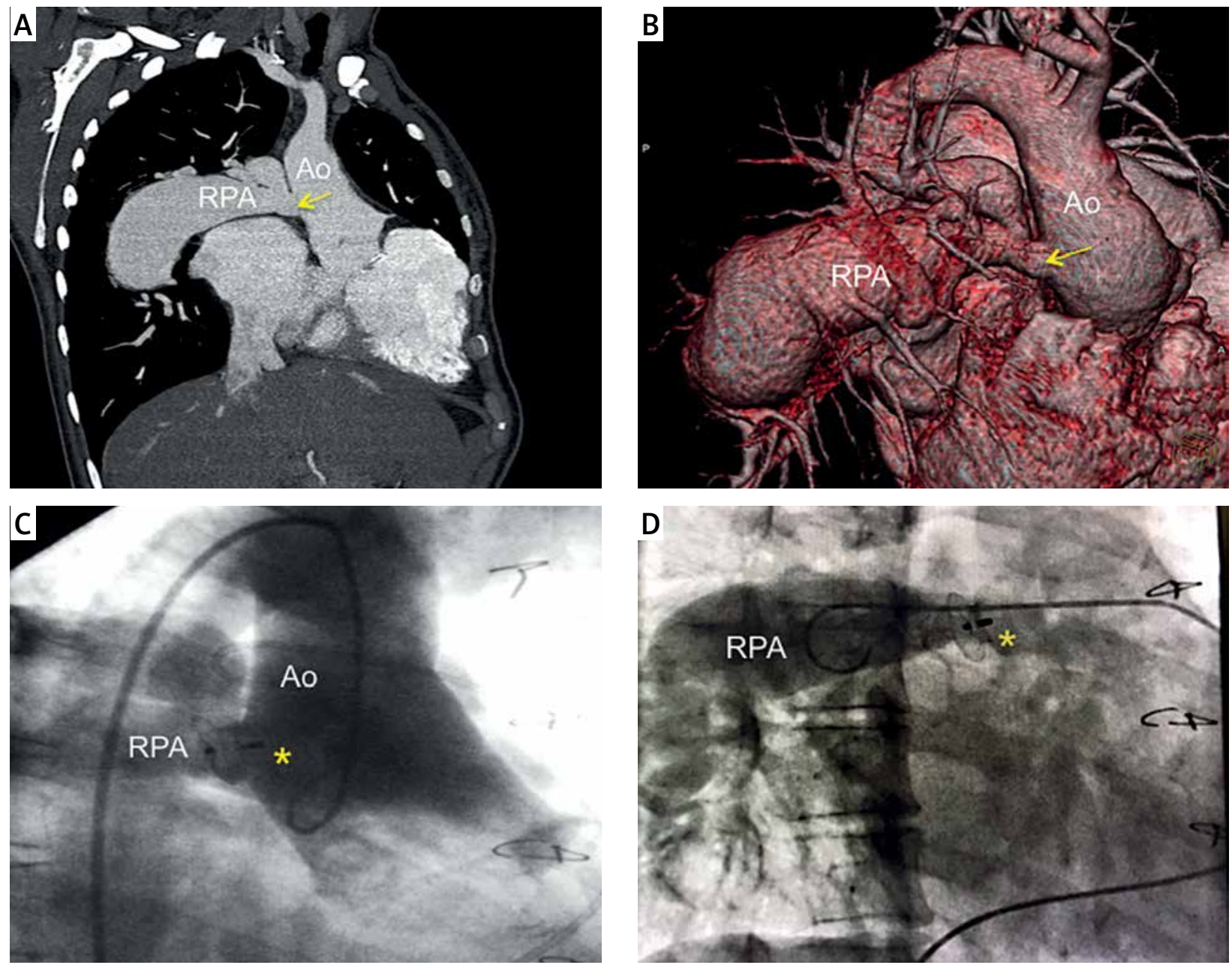

Figure 1. 2D (A) and 3D (B) CT angiogram features of the shunt between the ascending aorta (Ao) and right pulmonary artery (RPA) (yellow arrow). Angiographic results of percutaneous occlusion of aortic-pulmonary shunt with $12 \mathrm{~mm}$ Amplatzer MVSO (yellow asterisk) implanted from the arterial side, immediately after the intervention (C) and 5 years later (D)

\section{Conflict of interest}

The authors declare no conflict of interest.

\section{References}

1. Yuan SM1, Jing H. Palliative procedures for congenital heart defects. Arch Cardiovasc Dis 2009; 102: 549-57.

2. Goldberg DJ, French B, McBride MG, et al. Impact of oral sildenafil on exercise performance in children and young adults after the Fontan operation: a randomized, double-blind, placebo-controlled, crossover trial. Circulation 2011; 123: 1185-93.

3. Folino AF, Daliento L. Arrhythmias after tetralogy of Fallot repair. Indian Pacing Electrophysiol J 2005; 5: 312-24.

4. Llamas JP, Szkutnik M, Fiszer R, Białkowski J. Treatment of elevated pulmonary artery pressure in a child after Glenn procedure: transcatheter closure of pulmonary artery banding with subsequent sildenafil therapy. Kardiol Pol 2012; 70: 201-3. 